\title{
Human Herpesvirus 8-Unrelated Primary Effusion Lymphoma-Like Lymphoma in an Elderly Korean Patient with a Good Response to Rituximab Plus Cyclophosphamide, Doxorubicin, Vincristine, and Prednisolone
}

\author{
Junghoon Shin, $\mathrm{MD}^{1}$ \\ Jeong-Ok Lee, $M D^{1}$ \\ Ji-Young Choe, MD2 \\ Soo-Mee Bang, MD, PhD' \\ Jong-Seok Lee, MD, PhD'
}

Departments of ${ }^{1}$ Internal Medicine and ${ }^{2}$ Pathology, Seoul National University Bundang Hospital, Seongnam, Korea
Primary effusion lymphoma (PEL) is a rare type of non-Hodgkin's lymphoma arising from a B-cell lineage characterized by the formation of malignant effusion in body cavities without evidence of a detectable tumor. The effusion contains tumor cells universally infected with human herpesvirus 8 (HHV8), which is the critical factor differentiating PEL from HHV8-unrelated PEL-like lymphoma (PEL-LL). This report describes a 77-year-old male patient with pleural effusion and ascites, containing lymphoma cells expressing a B-cell phenotype, but without markers of HHV8 in immunocytochemical analysis. The patient was diagnosed with PEL-LL and treated with six cycles of rituximab plus cyclophosphamide, doxorubicin, vincristine, and prednisolone (R-CHOP), which resulted in a complete remission. The patient is currently disease-free 15 months post-treatment. To the best of our knowledge, this is the first report on administration of R-CHOP in a PEL-LL patient in South Korea.
Correspondence: Jeong-Ok Lee, MD Department of Internal Medicine, Seoul National University Bundang Hospital, 82 Gumi-ro 173beon-gil, Bundang-gu,

Seongnam 13620, Korea

Tel: 82-31-787-7055

Fax: 82-31-787-4098

E-mail: jeongok77@gmail.com

Received February 19, 2016

Accepted May 12, 2016

Published Online June 10, 2016
Key words

Primary effusion lymphoma, Human herpesvirus 8, R-CHOP protocol

\section{Introduction}

Primary effusion lymphoma (PEL) is a distinct type of nonHodgkin's lymphoma (NHL) presenting with a characteristic feature of pleural, peritoneal, or pericardial effusions without detectable tumor masses [1]. PEL is universally associated with the presence of human herpesvirus 8 (HHV8) within the neoplastic cells. Most cases are associated with immunodeficient states, particularly human immunodeficiency virus infection, which accounts for more than $75 \%$ of reported cases [2,3]. However, numerous cases of NHL with remarkable clinicopathologic similarities to PEL without evidence of HHV8 infection, and occasionally, without any factors predisposing to immunodeficiency have been reported [4-6]. This subset of cases, although rare, have been categorized separately and constitute a distinct disease entity referred to as HHV8-unrelated PEL-like lymphoma (PELLL). Differentiation of PEL-LL from PEL is important, as they appear to have distinct demographics, immunophenotypes, 
responses to treatment, and prognoses $[5,7,8]$. Herein, we report on an elderly male patient diagnosed with PEL-LL who received six cycles of rituximab plus cyclophosphamide, doxorubicin, vincristine, and prednisolone (R-CHOP) chemotherapy. To the best of our knowledge, this is the first report on administration of R-CHOP in a PEL-LL patient in South Korea.

\section{Case Report}

A 77-year-old male presented with abdominal distension and pitting edema of both lower limbs for 1 month. He had no complaints of respiratory symptoms, such as a cough, sputum, dyspnea, and no history of fever or chills. On examination, his abdomen was soft and distended, with shifting dullness on percussion, and decreased breath sounds were detected in the right lower lung field, without audible crack- les or wheezes on auscultation. He had grade 2 pitting edema of both lower extremities, and there was no evidence of hepatosplenomegaly or enlargement of superficial lymph nodes. The hemogram was unremarkable, although the serum lactate dehydrogenase (LDH) level was elevated to 393 IU/L (reference range, 100 to $225 \mathrm{IU} / \mathrm{L}$ ). The chest X-ray showed a large right-sided pleural effusion, and electrocardiogram and thyroid function tests were normal.

Both thoracentesis and paracentesis were performed. The pleural effusion contained 5,750/ $\mu \mathrm{L}$ of red blood cells (RBCs) and $2,990 / \mu \mathrm{L}$ of white blood cells (WBCs). In the differential cell count, $82 \%$ of the WBCs in the routine cell count had an atypical morphology. The results of the chemical analysis of the pleural effusion were as follows: protein $3,833 \mathrm{mg} / \mathrm{dL}$, glucose $13 \mathrm{mg} / \mathrm{dL}, \mathrm{LDH}$ 6,000 IU/L, and adenosine deaminase $310 \mathrm{IU} / \mathrm{L}$. The acid-fast bacilli smear and culture and real-time nested polymerase chain reaction for Mycobacterium tuberculosis complex DNA in both the pleural effusion and sputum showed negative results. No microorganisms were identified on Gram stain and from bacterial culture. Ascites
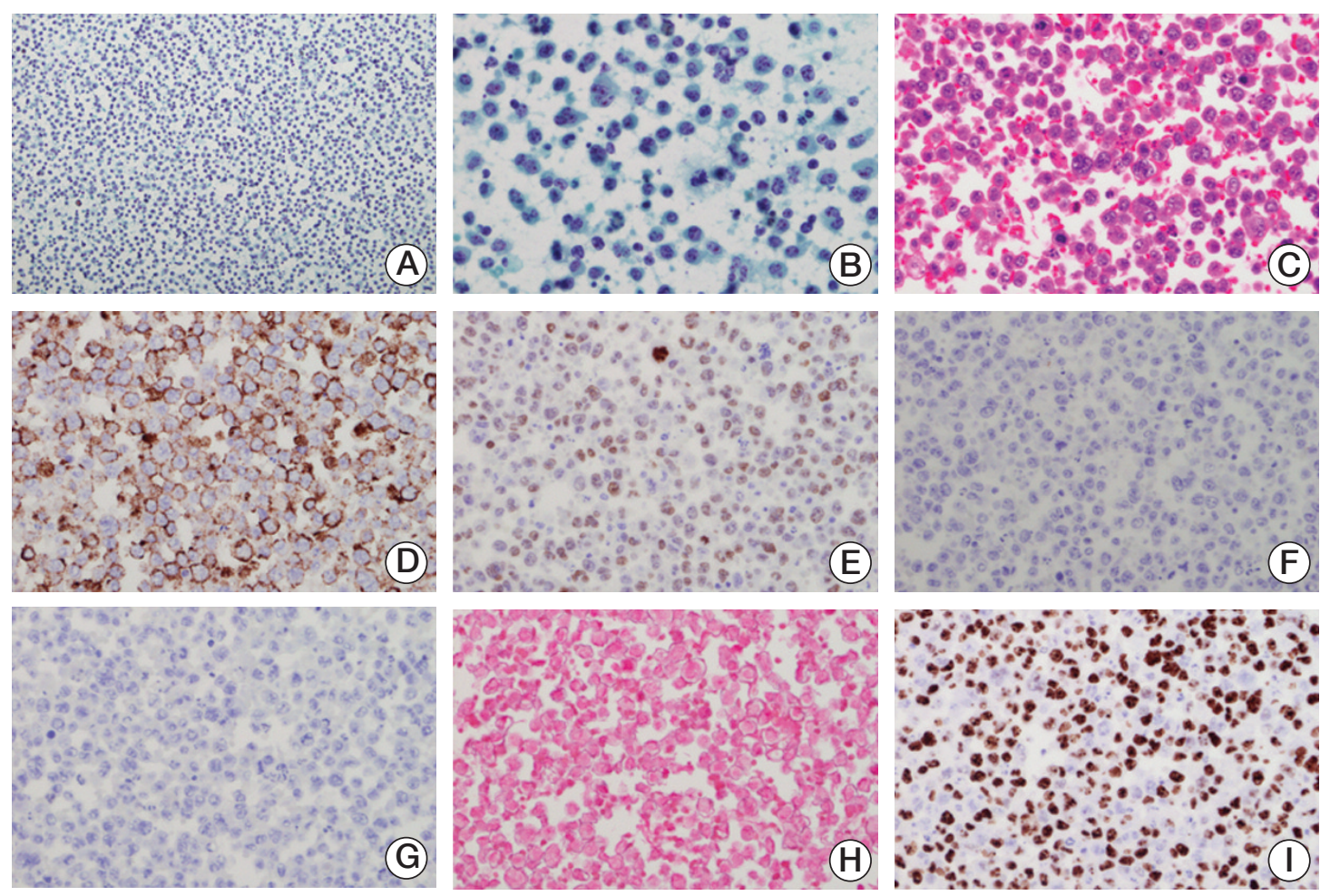

Fig. 1. Pleural effusion showing large and pleomorphic cells with immunoblastic or anaplastic features in cytospin or cell block preparation, which were positive for CD20 and MUM-1 with weak-to-moderate intensity, but negative for CD138, human herpesvirus 8 (HHV8), and Epstein-Barr virus-encoded small RNA, showing a high Ki-67 proliferating index: Papanicolaou (PAP) (×100) (A), PAP (×400) (B), H\&E (×400) (C), CD20 (D), MUM-1 (E), CD138 (F), HHV8 (G), Epstein-Barr virus $(\mathrm{H})$, and $\mathrm{Ki}-67(\mathrm{I})$. 

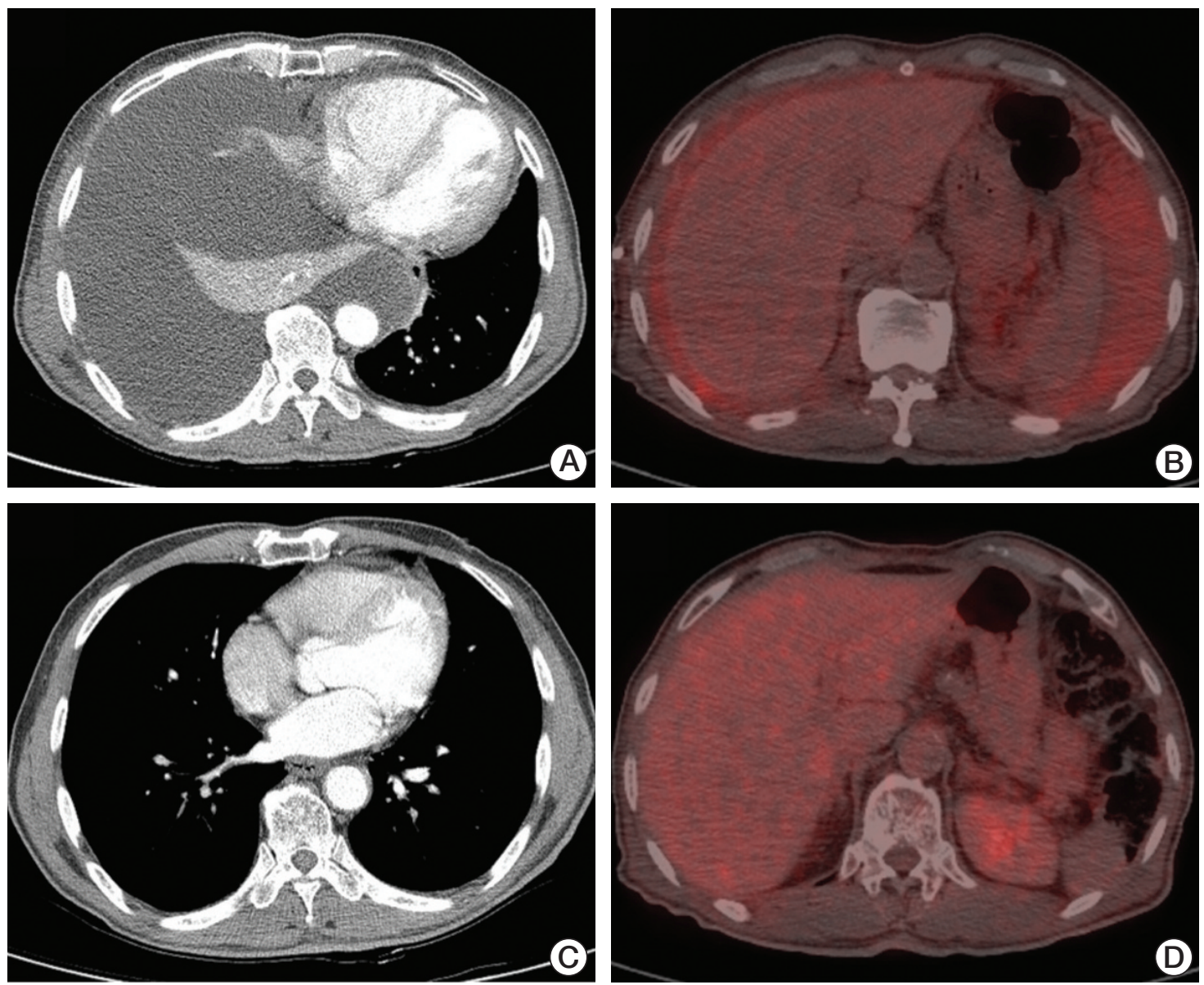

Fig. 2. Imaging study at the time of diagnosis and after completion of chemotherapy: computed tomography (CT) at diagnosis (A), positron emission tomography-computed tomography (PET-CT) at diagnosis (B), CT after six cycles of rituximab plus cyclophosphamide, doxorubicin, vincristine, and prednisolone (R-CHOP) (C), and PET-CT after six cycles of R-CHOP (D).

contained $200 / \mu \mathrm{L}$ of RBCs and $10,880 / \mu \mathrm{L}$ of WBCs with $33 \%$ lymphocytes and $67 \%$ atypical cells. Results of chemical analysis showed a total protein of $3,908.9 \mathrm{mg} / \mathrm{dL}$, albumin of $2,400 \mathrm{mg} / \mathrm{dL}$, glucose of $34 \mathrm{mg} / \mathrm{dL}$, and $\mathrm{LDH}$ of 3,476 $\mathrm{IU} / \mathrm{L}$. The serum-ascites albumin-gradient was $0.8 \mathrm{~g} / \mathrm{dL}$, and the Gram stain and bacterial cultures were negative.

The cytological evaluation of both the pleural effusion and ascites demonstrated numerous large and pleomorphic cells with eosinophilic macronucleoli and abundant cytoplasm, with an immunoblastic or anaplastic appearance (Fig. 1A-C). Tumor markers, including prostate-specific antigen (PSA), carcinoembryonic antigen, and carbohydrate antigen 19-9 were assessed in order to clarify the origin of the malignant effusion. Esophagogastroduodenoscopy (EGD), colonoscopy (CS), computed tomography (CT) of the chest, abdomen, and pelvis, whole body positron emission tomography-com- puted tomography (PET-CT), and bone scan were performed. The serum PSA level was increased to 7.713, while other tumor markers were within the normal range. EGD and CS detected some tubular adenomatous polyps, which were confirmed as benign. CT scans showed a large pleural effusion, contrast enhancement of the peritoneum, and prostatic enlargement (Fig. 2A). Therefore, a percutaneous drainage catheter was inserted into the chest, and a prostate biopsy was performed, which confirmed that the prostate tissue was benign. Diffuse hypermetabolism along the omental and peritoneal infiltration was detected on the PET-CT scan (Fig. 2B), and no significant abnormal uptake was detected on the bone scan.

On immunocytochemistry, the neoplastic cells in the pleural effusion were CD45-positive and cytokeratin-negative, and the tumor cells were positive for CD20 and MUM-1 with 
weak-to-moderate intensity and negative for CD138, CD79a, CD3, Bcl-2, Bcl-6, and HHV8. The Ki-67 proliferating index was approximately $90 \%$. Epstein-Barr virus (EBV)-encoded small RNAs in situ hybridization showed no EBV-infected cells (Fig. 1D-I).

Serological tests were negative for human immunodeficiency virus (HIV), hepatitis B virus (HBV), and hepatitis C virus (HCV) and positive for anti-hepatitis B surface antibody with a titer of $141.2 \mathrm{IU} / \mathrm{L}$, consistent with a history of HBV vaccination. Bone marrow examination detected hypocellular marrow (cellularity of 21\%-30\%) without evidence of lymphoma involvement, and cytogenetic examination detected a normal karyotype: $46, X Y$.

Based on the results described above, the patient was diagnosed with PEL-LL without concurrent HCV, HBV, or HIV infection. Six cycles of R-CHOP chemotherapy with symptomatic improvement was administered following the completion of therapy. The CT scan showed almost complete resolution of the pleural effusion and ascites with a decrease in the omental infiltration and peritoneal thickening with no metabolic activity on the PET-CT (Fig. 2C and D). The patient has been in a progression-free state for 15 months.

\section{Discussion}

Although PEL was first described in 1996 [9], the biological mechanism underlying the formation of a malignant effusion without detectable tumor masses is uncertain. This presentation is the most remarkable feature differentiating PEL from other types of conventional lymphomas. Development of PEL is provoked by HHV8 infection; however, the role played by HHV8 in lymphomagenesis remains to be clarified. PEL is one of the three major categories of HIV-associated NHL, accounting for approximately $1 \%-4 \%$ of HIVassociated NHL [2]. The diagnosis of PEL requires a cytological evaluation of the malignant effusion or the epithelial tissue lining the involved cavity, which shows large neoplastic cells with intermediate morphology between immunoblastic and anaplastic features $[4,10]$. These cells contain large nuclei with prominent nucleoli and variable amounts of basophilic cytoplasm. The immunocytochemical staining shows a lack of expression of pan-B-cell markers, including CD19, CD20, and CD79a. Universal expression of ORF73/latent nuclear antigen-1, the standard marker for detection of HHV8 infection, is required for making the diagnosis [3,4]. PEL is distinguished from Burkitt's lymphoma by its lack of c-myc proto-oncogene rearrangement [9]. In a recent cohort study, Notch1, a protein involved in a transmembrane signal transduction pathway, was proposed as a potential effector pro- tein associated with the oncogenic process in HHV8-infected cells [3]. Although few studies describing the natural course in this subset of patients with PEL have been reported, the prognosis is considered extremely poor, and no optimal treatment has yet been established [10], with a median overall survival of less than 6 months [11]. Although few studies to identify prognostic factors associated with survival in patients with PEL have been reported, a retrospective literature review suggested a close association of the number and location of involved body cavities with overall survival [11].

The microscopic morphology of PEL-LL is similar to that of conventional PEL; however, there are several important differentiating factors. For example, PEL-LL is mainly associated with expression of conventional B-cell-markers, including CD19, CD20, and CD79a, usually occurs in older patients without concurrent immunocompromised conditions, such as HIV infection or organ transplantation, and, as its name implies, is not connected with HHV8 infection. PELLL is usually not accompanied by a viral infection; thus, its oncogenic mechanism is not well understood. Contrary to what is observed with PEL, PEL-LL cells usually carry c-myc amplification and show Burkitt-like morphology $[9,11,12]$. Therefore, lymphomagenesis is thought to involve multi-step genomic abnormalities. According to the most recent data, patients with PEL-LL show a better response to chemotherapy and a longer survival than those with PEL, with a median survival of more than 10 months compared with less than 6 months, respectively $[9,13]$. Despite the similarity in clinical presentation, it is unknown why PEL-LL has a better prognosis than PEL.

To date, approximately 50 cases of PEL-LL have been reported in the literature [7,13-15]. Most cases were HIV negative, and a small percentage were EBV and $\mathrm{HCV}$ positive, accounting for approximately $20 \%$ and $40 \%$ of cases, respectively [6]. In patients with positive serology for $\mathrm{HCV}$, persistent antigenic stimulation of the virus is potentially responsible for the oncogenic process. However, a substantial number of cases without evidence of viral infection have been reported. More studies investigating the clonal origin of neoplastic cells and possible genetic precipitating factors are necessary in order to determine the mechanism of lymphomagenesis in such cases. Although the optimal therapeutic strategy is unclear, CHOP-based chemotherapy with or without rituximab has been used in a substantial number of cases [13]. In a previous literature review, the mean survival time for patients treated with CHOP or R-CHOP was 16.3 months ( $\mathrm{n}=22)$, but only 12.5 months for patients without treatment $(n=10)$ [13]. Several other treatment approaches have also been proposed in case reports or in small case series. One such example is a combination of rituximab and oral sobuzoxane and etoposide, which showed a promising result according to a Japanese case report [12]. 
Reports regarding either PEL or PEL-LL are rare in Asia. The first case report of a Korean PEL-LL patient was an 80year-old female diagnosed with PEL-LL after treatment for tuberculous pleurisy who survived a long time without chemotherapy [15]. Our case demonstrated the typical clinical features of PEL-LL described in previous reports: the patient was elderly with no evidence of HBV, HCV, or HIV infection; the cancer cells expressed conventional B-cell markers including CD20; R-CHOP chemotherapy resulted in a good response, consistent with the earlier finding that chemotherapy is more effective for treatment of PEL-LL than PEL. However, considering the long survival of the patient in the previously reported case who was not treated with any anti-cancer agents, the role of chemotherapy in PEL-LL is unclear. Therefore, to establish practical guidelines for the treatment of PEL-LL, randomized trials with a large cohort are required to compare the efficacy of various therapeutic approaches.

\section{Conflicts of Interest}

Conflict of interest relevant to this article was not reported.

\section{References}

1. Chen YB, Rahemtullah A, Hochberg E. Primary effusion lymphoma. Oncologist. 2007;12:569-76.

2. Little RF, Gutierrez M, Jaffe ES, Pau A, Horne M, Wilson W. HIV-associated non-Hodgkin lymphoma: incidence, presentation, and prognosis. JAMA. 2001;285:1880-5.

3. Wang HY, Fuda FS, Chen W, Karandikar NJ. Notch1 in primary effusion lymphoma: a clinicopathological study. Mod Pathol. 2010;23:773-80.

4. Carbone A, Gloghini A. PEL and HHV8-unrelated effusion lymphomas: classification and diagnosis. Cancer. 2008;114: 225-7.

5. Alexanian S, Said J, Lones M, Pullarkat ST. KSHV / HHV8-negative effusion-based lymphoma, a distinct entity associated with fluid overload states. Am J Surg Pathol. 2013;37:241-9.

6. Mohammad F, Siddique MN, Siddiqui F, Popalzai M, Asgari M, Odaimi M. A unique case of malignant pleuropericardial effusion: HHV-8-unrelated PEL-like lymphoma: a case report and review of the literature. Case Rep Oncol Med. 2014;2014: 436821.

7. Fan HB, Yang DL, Guo Y, Chen AS, Zhou MX, Wu JJ, et al. Human herpes virus 8-unrelated primary effusion lymphomalike lymphoma in a patient with hepatitis B virus-related liver cirrhosis: a case report. J Res Med Sci. 2014;19:190-2.

8. Kashiwagi T, Minagawa K, Kawano H, Hirata T, Kashiwagi S, Nakagawa Y, et al. HIV-negative, HHV-8-unrelated primary effusion lymphoma-like lymphoma with genotypic infidelity and c-MYC expression. Ann Hematol. 2014;93:1609-10.

9. Nador RG, Cesarman E, Chadburn A, Dawson DB, Ansari
MQ, Sald J, et al. Primary effusion lymphoma: a distinct clinicopathologic entity associated with the Kaposi's sarcomaassociated herpes virus. Blood. 1996;88:645-56.

10. Okada S, Goto H, Yotsumoto M. Current status of treatment for primary effusion lymphoma. Intractable Rare Dis Res. 2014;3:65-74.

11. Castillo JJ, Shum H, Lahijani M, Winer ES, Butera JN. Prognosis in primary effusion lymphoma is associated with the number of body cavities involved. Leuk Lymphoma. 2012;53: 2378-82.

12. Toyoda K, Abe Y, Tsuda M, Haji S, Choi I, Suehiro Y, et al. Successful treatment with oral low-dose sobuzoxane and etoposide combined with rituximab in an elderly patient with HHV-8-negative primary effusion lymphoma-like lymphoma. Rinsho Ketsueki. 2014;55:815-9.

13. Wu W, Youm W, Rezk SA, Zhao X. Human herpesvirus 8-unrelated primary effusion lymphoma-like lymphoma: report of a rare case and review of 54 cases in the literature. Am J Clin Pathol. 2013;140:258-73.

14. Wu W, Liu J, Hong W. Human herpes virus 8-unrelated primary effusion lymphoma-like lymphoma diagnosed by fluorodeoxyglucose positron emission tomography/computer tomography and laparoscopy. Oncol Lett. 2014;7:433-4.

15. Kim KH, Lee JH, Jeong HC, Kim GW, Song SH, Jung SY, et al. A case of human herpes virus- 8 unrelated primary effusion lymphoma-like lymphoma presented as pleural effusion. Tuberc Respir Dis. 2012;73:336-41. 